\title{
Simulation and control of multipurpose wheelchair for disabled/elderly mobility
}

\author{
N.M. Abdul Ghani ${ }^{\mathrm{a}, *}$ and M.O. Tokhi ${ }^{\mathrm{b}}$ \\ ${ }^{a}$ Faculty of Electrical and Electronics Engineering, Universiti Malaysia Pahang, Pekan, Malaysia \\ ${ }^{\mathrm{b}}$ Deparment of Automatic Control and Systems Engineering, The University of Sheffield, Sheffield, UK
}

\begin{abstract}
This paper presents investigations into the development of modelling and control strategies for a multipurpose wheelchair as mobile transporter for elderly and disabled people. The research is aimed at helping people with physical weakness/disabilities in their upper and lower extremities to move independently without human intervention. A novel reconfiguration which allows multi-task operations in the same wheelchair system with improved design is modelled in Visual Nastran 4D (VN4D) software. A modular fuzzy logic control mechanism with integrated phases is introduced for the overall operations and two-wheeled stabilization of the wheelchair. It is shown that the proposed modular fuzzy control approach is able to ensure system stability while performing multipurpose tasks such as manoeuvrability on flat surfaces, stairs climbing (ascending and descending), standing in the upright position on two wheels and transformation back to standard four wheels with up to $50 \%$ less initial torque in comparison to previous designs.
\end{abstract}

Keywords: Multipurpose wheelchair, stair climbing, sit-to-stand, stand-to-sit, modular fuzzy logic control

\section{Introduction}

The current worldwide trend in increased disabled and elderly population has challenged extensive designs and advancements in mobility transport as essential needs. This includes mobility devices such as wheelchairs, which vary in designs depending on their functionalities, from use in sports for Paralympics or other sportspersons to individual use for outdoor and indoor environments. It is vital to an individual who uses the wheelchair as the main self-mobility transport to move from one place to another independently. Currently, standard four-wheeled wheelchair designs have some limitations and cannot perform standard routine tasks, such as stair climbing, sit-to-stand and stand-tosit operations.

A stair climbing wheelchair will allow the user to utilise the same assistive mobility equipment to ma-

${ }^{*}$ Corresponding author: N.M. Abdul Ghani, Faculty of Electri$\mathrm{cal}$ and Electronics Engineering, Universiti Malaysia Pahang, 26600 Pekan, Malaysia. Tel.: +60 609 4246087; Fax: +60 609 4246111; E-mail:normaniha@ump.edu.my. noeuvre on stairs as well as on flat surfaces. There will be no need for an elevator or an assistant to perform stair climbing, and this will allow the wheelchair user to exercise independence. A significant amount of work has been reported in the literature on the development and control of stair-climbing wheelchairs. These include crawler type [25,27,39,41,42], wheeled type [24,33,37] and legged type [29] wheelchairs.

The crawler type wheelchair works well on an uneven terrain and provides a high terrain adaptivity. The first commercial wheelchair models were based on a single-section track mechanism capable of climbing up and down staircases $[39,42]$. The design of the overall stair climber mechanism needed more refinement so as to reduce its total weight. Moreover, crawler mechanisms have some drawbacks when stepping on the edge of the first step; the entire track is forced to rotate during climbing down a staircase [27].

Nakajima proposed a step-up gait called RT-Mover [29], which comprised a four-wheel-type mobile robot for upward step like a legged robot with simple leg mechanism. The robot can move like a wheeled robot on normal terrain and transform to legged mechanism 
during climbing a step. The wheel is lifted like a leg while supporting the body on at least three points in order to remain level on a supporting polygon. However, due to limitation in the robot's leg height it can perform step climbing tasks only on step dimensions with stair riser heights of $0.1 \mathrm{~m}$ and long stair tread depths for each step. Moreover, this robot does not seem practical for use in places where there are more than two steps and if the stairs' slope is high.

A noteworthy commercialized iBOT wheelchair was introduced in the early $1990 \mathrm{~s}$ to perform balancing and mobility in two-wheeled configuration and in stair climbing tasks [21]. The system only relies on the changes of centre of gravity (COG) as input signal in order to move from one step to another. In order to achieve that, the user needs to tilt the back seat forward and backward while holding the stair handrail to perform ascending and descending on stairs respectively $[12,13]$. Thus, it requires strong hand muscle to hold the handrail and support the whole wheelchair system on the stairs. Moreover, it requires another person or assistance to tilt the back seat if the disabled/elderly person is unable to use her/his own hands.

Disabled people using wheelchairs are exposed to many health problems associated with being sedentary. These include physical and psychological effects due to prolonged seated posture [17]. Other effects include depression and loss of confidence, which often result from feelings of loss of control in certain situations. A study to examine how frequently healthy free-living adults perform sit-to-stand movement on daily basis has been conducted recently and the results show that the participants perform on average $60( \pm 22)$ movements each day [31].

Thus, there is a need for sit-to-stand mechanism so that the disabled users can boost their confidence level and gain the associated health benefits. For example, standing maintains leg muscles in reasonable physical condition, improves blood circulation, urinary health and bowel function, and reduces spasticity and pressure sores efficiently [30]. Many paraplegics utilise walker type and exoskeleton devices as assistive devices using robot technology. For example, braces and crutches have been widely used by people with spinal cord injury (SCI) to provide the opportunity to stand and move. A standing-up robotic supportive device has been used to raise the human from sitting to standing posture. The device is driven by an electrohydraulic servo system and supports the subject under the buttocks [23]. Authors in [20] integrated functional neu- romuscular stimulation (FNS) with braces to activate sensory neurons in paralyzed muscles using electrical stimulation to control standing posture. However, the system needs an assistant to support the standing process and overall stability. Moreover, current designs of such devices have several limitations; they are bulky and hard to manoeuver in narrow spaces, are heavy and require considerable user's physical effort, thus increasing fatigue $[23,30]$.

A design enabling the wheelchair to transform from four wheeled to two wheeled mode in an upright position, lift the seat to a higher position, perform linear motion and allow transformation back from two-wheeled to four-wheeled mode has been reported in [4-7,21]. The two-wheeled wheelchair system uses the same concept as a double inverted pendulum, which is known to be unstable as it needs to take into account the whole weight of the system and the user $[5,6]$. Moreover, recently a novel two-wheeled configuration vehicle has been reported [8], which has shown promising stability features when balancing a payload with a movable payload actuator placed on the second pendulum link. The authors have used the two link inverted pendulum concept for lifting both the front wheels and stabilizing the overall wheelchair body on its rear wheels in the upright two-wheeled mode. However, the system does not have standing mode and associated motion capability. The humanoid that was used as load was rigidly attached to the seat throughout the operations without any changes to its dynamics. Moreover, the wheelchair produced initial high torque at the wheels and tilt motors (Link 1 and Link 2) to lift the overall mechanism to the twowheeled upright position. This will produce large tilt angles at the beginning of the transformation process as the overall system will need to be tilted with such high power [4-7]. The comfort issue of the user is important in this case especially for a disabled/elderly person.

The current research focuses on development of a novel compact and light weight multipurpose wheelchair model and further on the implementation of modular fuzzy logic control (FLC) strategies to perform the corresponding multipurpose tasks. Intuitively, although all the classical control methods have shown promising results in performing two-wheeled stability function, there are nonlinearity and uncertainty issues that need to be considered. Although fuzzy logic has been used intensively in both single and double inverted pendulum systems $[18,26,32,38,44,49,50]$, none has implemented the approach in stair climbing wheelchair with 
the inverted pendulum concept. In order to cater for the inherent nonlinearity of the two wheeled system, an intelligent control approach based on fuzzy logic is investigated in this work $[2,3,22,35,36,50-56]$. This research embarks on the development of an automatic interchangeable phases control structure using a modular fuzzy logic (IPFL) approach to coordinate the overall operations and to ensure system stability and safety.

It is aimed to help people with disability/weakness in their lower extremities, so to enable them manoeuvre independently [41]. The wheelchair prototype presented in this work adopts a similar mechanism as the commercial iBOT and NOBOROT, and previous work [4-7] but with the addition of important properties such as capability to perform stair climbing (ascending and descending) and standing/sitting motions with reduced initial torque and reduced tilt angle within the same wheelchair system.

The rest of the paper is organized as follows: Section 2 introduces the wheelchair design in Visual Nastran software. Section 3 proposes the modular FLC strategy for multipurpose tasks. Section 4 presents simulation results on performance assessment of the proposed control algorithm. Section 5 concludes the paper.

\section{Multipurpose wheelchair model}

Mathematical modelling of the wheelchair configuration to achieve multi-tasking is quite complex. Several assumptions have to be made for purposes of simplicity when deriving the corresponding complex and nonlinear mathematical equations. Thus, some considerations and important features might be left out due to the linearization process of the mathematical model. A simplified version of mathematical model is not sufficient to investigate the whole system performance due to the complex nature of the multipurpose wheelchair system. Therefore, in order to preserve the nonlinear aspect, a new reconfigurable wheelchair and humanoid model are developed in Visual Nastran 4D (VN4D) environment which allows assembling 3D parts and combining all physical interactions in a computer aided design (CAD) model $[28,45]$. It gives performance characteristics close to that of the real system through finite element analysis (FEA); motion and collisions, vibration, friction and gravity effects. Moreover, it is equipped with controllable elements such as sensors and meters that can be linked to Simulink/Matlab for developing and evaluating designed controllers. The

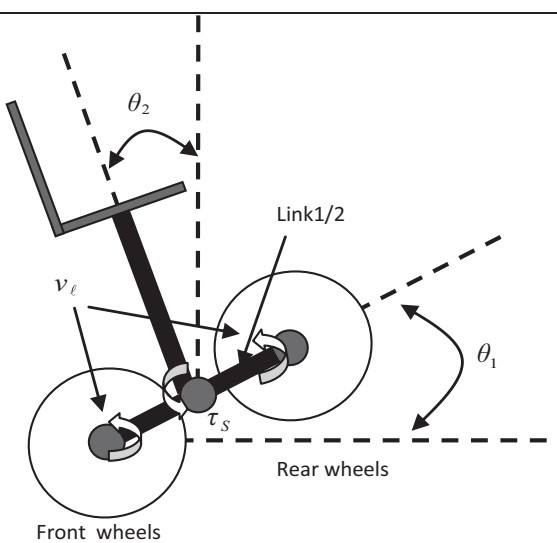

(a)

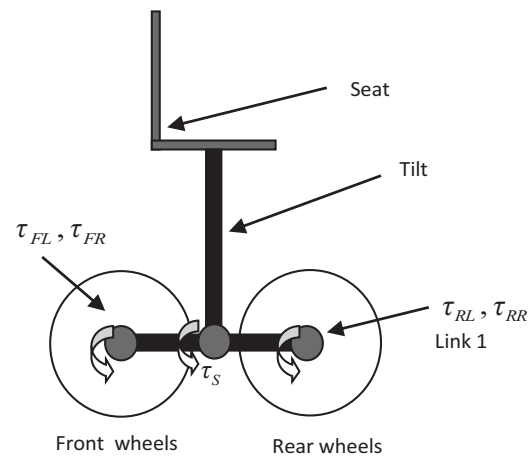

(b)

Fig. 1. Schematic diagram of a wheelchair in (a) standard mode (b) two-wheeled mode.

VN4D software is used as a simulation platform for testing the control action and the whole system simulation before it can be implemented in real hardware.

In this work, a cluster/link rotation mechanism is utilized with separate motors for the wheels and cluster/link control to perform multi-tasking work including stair climbing and sit-to-stand/stand-to-sit motions. Moreover, it allows for more degrees of freedom for the wheelchair system so that the wheels motors can be used to control the position, while the cluster/link motor to control the link rotation for the climbing operation.

\subsection{Stair climbing}

A detailed schematic diagram of the wheelchair mechanism is shown in Fig. 1 in two dimensions in standard four-wheeled mode. Note that each wheel is driven by a motor; $\tau_{F L}, \tau_{F R}$ represent the torques due to left and right front wheel motors, and $\tau_{R L}, \tau_{R R}$ are torques due to the left and right rear wheel motors 


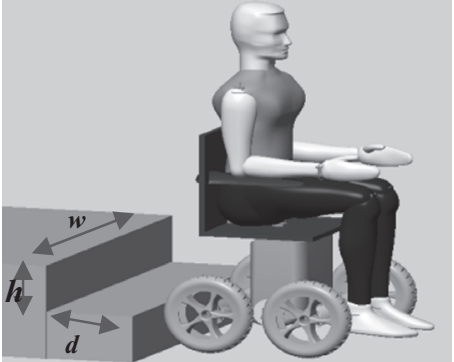

Fig. 2. Standard mode.

respectively. The climbing mechanism involves two links, Link1 and Link 2, placed at the right and left side of the wheelchair axle respectively. In order to perform a climbing task, both links need to be rotated by the link/cluster motor, $v_{\ell}$, using the link rotation mechanism. The link/cluster motor, $v_{\ell}$ is placed at both left and right links for the link/cluster rotation, angle $\theta_{1}$, to perform the two-wheeled stabilization and climbing tasks. In this research, a human model with $1.75 \mathrm{~m}$ height and $71 \mathrm{~kg}$ weight as an adult average height/weight is used. However, the tolerance level of the human's weight is $\pm 10 \mathrm{~kg}$ for the system around the designed weight, and so the system will be able to perform in a stable manner for up to $81 \mathrm{~kg}$ human weight. The height and sex of the user would not cause a problem as long as the weight is within the given range. During this operation, the seat which carries a humanoid with a weight of $71 \mathrm{~kg}$ might oscillate forward and backward due to the cluster rotation of the wheelchair. Thus, it is important that the seat is in the upright position, $\theta_{2}$ at all times with the seat torque, $\tau_{s}$. In this design, the seat is attached to a revolute motor for the control actuation.

The wheelchair parameters were taken from standard wheelchair dimensions [5,6], and the wheels dimensions were adopted from the iBOT mobility system which conforms to the applicable requirements of the ISO 7176-19: 2008 standard [46].

Figure 2 shows a straight stairway that was tested in VN4D simulation where each flight of stairs continues in the same direction as the previous flight. According to codes of practice and standards, the recommended range for a stair slope is from $30^{\circ}$ to $35^{\circ}$ due to its preference by people. However, the maximum range is $20^{\circ}-50^{\circ}$. A straight stairway which follows the Canadian Centre for Occupational Safety and Health, 2010 recommendation [9] is tested in this work with a slope of $31.5^{\circ}$ and steps with height, $h$ and width, $w$ as illustrated in Fig. 2. The design adheres to standard dimensions and structure to allow safe manoeuvrability and
Dimension and specifications of the wheelchair and humanoid model

\begin{tabular}{lll}
\hline Item & Dimension $(\mathrm{m})$ & Mass $(\mathrm{kg})$ \\
\hline Wheelchair body & & \\
Wheel & $0.15 \times 0.07$ & $1.50 \times 4$ \\
Seat & $0.45 \times 0.43 \times 0.08$ & 0.20 \\
Back rest & $0.02 \times 0.40 \times 0.45$ & 0.30 \\
Front horizontal axis & $0.04 \times 0.55$ & 14.00 \\
Back horizontal axis & $0.04 \times 0.55$ & 14.00 \\
Base link & $0.38 \times 0.06 \times 0.04$ & 1.00 \\
Left connecting rod & $0.34 \times 0.02 \times 0.01$ & 3.00 \\
Right connecting rod & $0.34 \times 0.02 \times 0.01$ & 3.00 \\
Left base joint & $0.05 \times 0.02$ & 1.00 \\
Right base joint & $0.05 \times 0.02$ & 1.00 \\
Vertical rod & $0.03 \times 0.45$ & 1.00 \\
Battery & $0.38 \times 0.23 \times 0.32$ & 1.55 \\
& &
\end{tabular}

feasibility of the wheelchair system as it is inherently hazardous. Two different tread sizes $d$ were tested in the simulations; $0.302 \mathrm{~m}$ and $0.407 \mathrm{~m}$. These parameters were chosen because $0.302 \mathrm{~m}$ is the minimum stair tread size that the wheelchair can climb as it is approximately equal to the wheel diameter. The stair tread size of $0.402 \mathrm{~m}$ was tested for the purpose of comparison with a different dimension following the ISO 7176-24: 2004 standard for requirements and test methods for user-operated, stair-climbing devices [47]. There is no maximum width, $w$ of the stairs as the minimum is set to $0.762 \mathrm{~m}$ to test the wheelchair in confined spaces. The wheelchair is able to perform stair climbing with the height, $h$ of up to $0.302 \mathrm{~m}$ which is approximately equal to the wheel diameter. The same limitation applies to the minimum stair's depth as studied in this work. Details of masses of the wheelchair components are given in Table 1. The humanoid was downloaded from a web site in a Solidwork format [10] and reconfigured in VN4D based on anthropometric data with height, $h_{b}$ and weight, $w_{b}$ [48]. These segment proportions serve as good approximations in the absence of better data or directly measured data from the individual. Recent works using similar VN4D environment for control system design purposes include $[1,5,6]$.

\subsection{Sit-to-stand and stand-to-sit}

The wheelchair model in standing position is shown in Fig. 3. This process requires a motor to provide rotation to a certain angle and remain stable while the system is in standing position (as used in previous task). A linear actuator and two revolute motors are added and introduced to fulfil the sit-to-stand transformation process. The linear actuator allows the human's leg to be placed on the leg rest while in the upright position, see Fig. 4(c). 


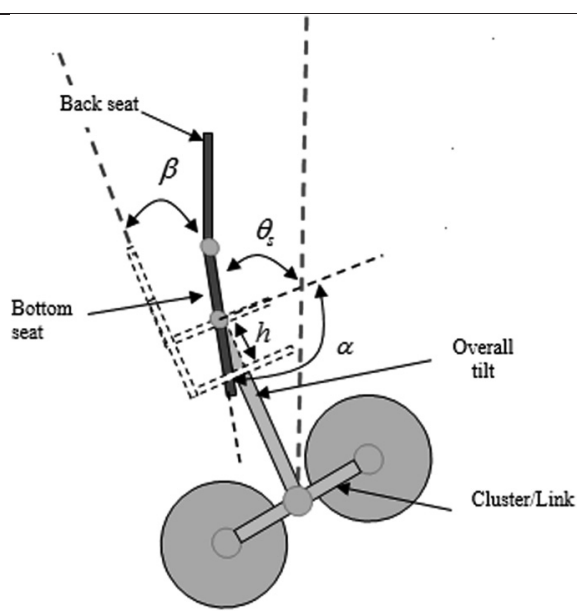

Fig. 3. Standing mechanism.

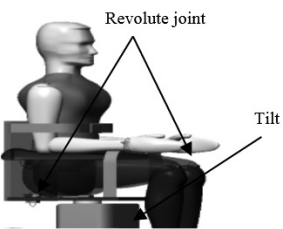

(a)

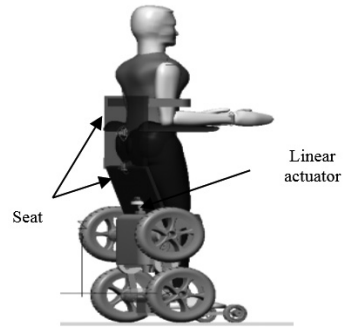

(c)

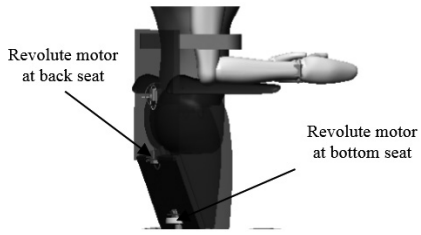

(b)

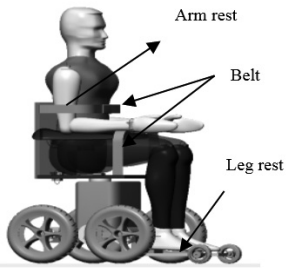

(d)
Fig. 4. Sit-to-stand and stand-to-sit.

Other revolute motors are added to the bottom seat and back seat of the wheelchair respectively as shown in Fig. 4(b). Revolute motors are placed at both bottom and back of the seat to allow the rotational operation to certain angles to realise the standing operation, in which case the back seat is kept at zero degree upright position.

In this work, the whole human body is not rigidly attached to the seat as it is made to be flexible to perform sit-to-stand and stand-to-sit motions. For this case, the lower part of human's body is connected to revolute joint that can rotate around the $y$-axis to allow the standing and sitting operations. The revolute joint is placed between trunk-thigh, and thigh-bottom leg for both left and right sides as shown in Fig. 4(a). This has increased the degree of freedom and the system tends to be unstable while performing the sit-to-stand and stand-to-sit motions due to free movement at the bottom part of human's body.

However, the trunk is rigidly attached to the back seat in order to hold the whole body while the leg rest is provided to support the human's weight. The same rigid joint is applied to the rest of the human's body connection.

For purposes of safety, a belt and knee stop are inserted at the upper and lower back seat to hold the trunk and legs from collapsing forward during the transformation process. Both facilities provide straps around the abdomen and knee which can be clipped or unclipped by the user, see Fig. 4(d). An arm-support mechanism is attached to both sides of the back seat for full support of user's arm while performing a standup operation. Authors in [15] have suggested that applying strong arm-support may lead to better transition from sitting to standing and the transformation becomes more stable. A meter (sensor) is attached to the wheelchair seat in order to feed the measured signal back to the controller for the linear actuation motor. The reference height for the human to stand on the leg rest is approximately $0.51 \mathrm{~m}$. The linear actuator is declared as the controller to produce the required control output to compensate for the height error. In this case, the control output signal is measured in velocity $(\mathrm{m} / \mathrm{s})$ and this will determine whether the actuator should increase or decrease its speed due to the input. The output of this linear actuator is seat height (m).

\section{Interchangeable phase modular fuzzy logic control structure}

Linear controllers such as PID or linear quadratic regulator (LQR) were not tested in this project as these have been considered in previous studies. The results have shown that fuzzy logic control outperforms PID and LQR in simulation [6] and hardware implementation [40]. A method of COG adjustment by the user input has previously been developed for stair climbing, in which the user or an assistant needs to adjust the COG of the wheelchair by leaning the wheelchair seat backward or forward depending on ascending or descending motion [12,13]. Moreover, in such a system, there is no stability assessment in ascending/ descending stair operation as the user will need to hold the handrail/guardrail at all times or rely on an assistant to keep the wheelchair system on the stairs from slipping off. An automatic stair climbing opera- 
tion for a wheelchair system provides extra advantages over such a method. In the current work, the transition from one step to another is done automatically using FLC. Therefore, an automatic mechanism using interchangeable phase modular fuzzy logic (IPFL) is designed to perform two tasks: 'balancing and stabilizing' and 'front and rear' wheels switching. A switching function is incorporated in the algorithm, which coordinates the switching between these two phases. Distance sensors are installed at both front and rear wheels to detect the required distance and this measurement $\mathrm{s}$ used for the switching.

The flow chart of switching operation is illustrated in Fig. 5(a) while Fig. 5(b) shows the specific coordination of both front and rear wheels motor torques, $\tau_{F}$ and $\tau_{R}$. This operation needs an integration between 'front and rear' link motors and 'front and rear' wheels motors which are highly interconnected to each other. In this case, IPFL is introduced which involves 'stabilizing and landing' phases and 'front and rear' wheels motor switching action. The stabilizing phase involves transformation from standard four wheeled to two wheeled mode in the vertical position. Once the system is in two wheeled mode, it is able to perform linear motion task according to the stairs tread dimension before landing. Several switches are used at the supervisory level to coordinate the decision making process for the climbing task. Accordingly, each switch shown in Fig. 5(b) will permit only one input at a time (either from point 1 or point 3 ) to pass through to the output. In this case, it acts as a coordinator selecting the required phase and wheel as input to the wheelchair system. The decision is based on a threshold condition located at point 2; if the threshold condition is met, the signal from point 1 is activated, otherwise the input signal from point 3 will be activated. The parameters ' $i l a$ ' and ' $i l b$ ' are the input signals to the fuzzy logic controller block, where FLC1a and FLC1b are for stabilizing and landing phases respectively, depending on state of switch1. The output of switch1 is link velocity, $v_{\ell}$ and acts as the control input signal to the system while the control output signal, angular position of link, is fed back to the system and establishes the state of switch 1 at point 2 .

A similar structure is used for the front and rear wheels motor switching operation, which involves FLC2 $a$ and FLC2b for control of the front and rear wheels respectively. The inputs to the fuzzy module block are a constant value of front wheels position, ' $i 2 a$ ', and rear wheels position, ' $i 2 b$ ', while $x_{1}$ and $x_{2}$ are feedback signals of these positions. The condition at point 2 is defined by the distance $d$ between respective wheels and stairs. Figure 5(b) shows detailed illustration of the specific 'front and rear' wheels motor switching coordination. It shows that there is a coupling effect between the distance of the wheels and the stairs for the front and rear side. This condition in this case is monitored by a supervisor to authorize the decision making process at Switch2_i, Switch2_ii, Switch2_iii and Switch2_iv which involves tight interconnections for the front and rear wheels torque switching action. There is no switching coordination for the seat motor torque, $\tau_{S}$, as the wheelchair system remains stable at constant vertical position, ' $i 3$ ' at all times throughout the overall climbing operation with $\theta_{2}$ as the feedback tilt angle.

The modular strategy is chosen to segregate complex tasks and to simplify the overall process while maintaining stability of the wheelchair system. In conventional FLCs, the number of rules increases exponentially as the number of system variables increases [34]. Modular FLCs (MFLC) can be used to reduce the number of rules by dividing a global task into sub-tasks independently and coordinating the subcontrollers to achieve the global objective. The modules are relatively autonomous and may interact with each other. The modular system is decomposed into $\mathrm{N}$ subsystems, located at the lower level. Each subsystem executes its own control law and communicates relevant information to the coordinator at the upper level [43]. The approach is selected here due to its ability to perform tasks in parallel for both stabilizing the two-wheeled system and maintaining wheelchair position. The operation of each fuzzy module is discussed in detail in the subsections below.

In the FLC, the inputs $e$ and $\Delta e$ with their respective gains are mapped onto a fuzzy inference to give a control output. Each module is designed with PDfuzzy Mamdani type and consists of five membership function (MF) levels; Positive Big (PB), Positive Small (PS), Zero (Z), Negative Big (NB) and Negative Small (NS). The inputs and outputs used result in $5 \times 5=$ 25 rules for each fuzzy controller with $25 \%$ overlap between the MFs. They are normalized at universe of discourse within the range $[-1,+1]$. Gaussian Bellshape MFs are used and this function is chosen to give smooth and steady response to the system.

A hybrid of proportional and derivative gain is integrated with fuzzy controllers to form PD-fuzzy control as shown in Fig. 6. This combination is chosen because the proportional and derivative terms can minimize the rise time and the steady state error respec- 


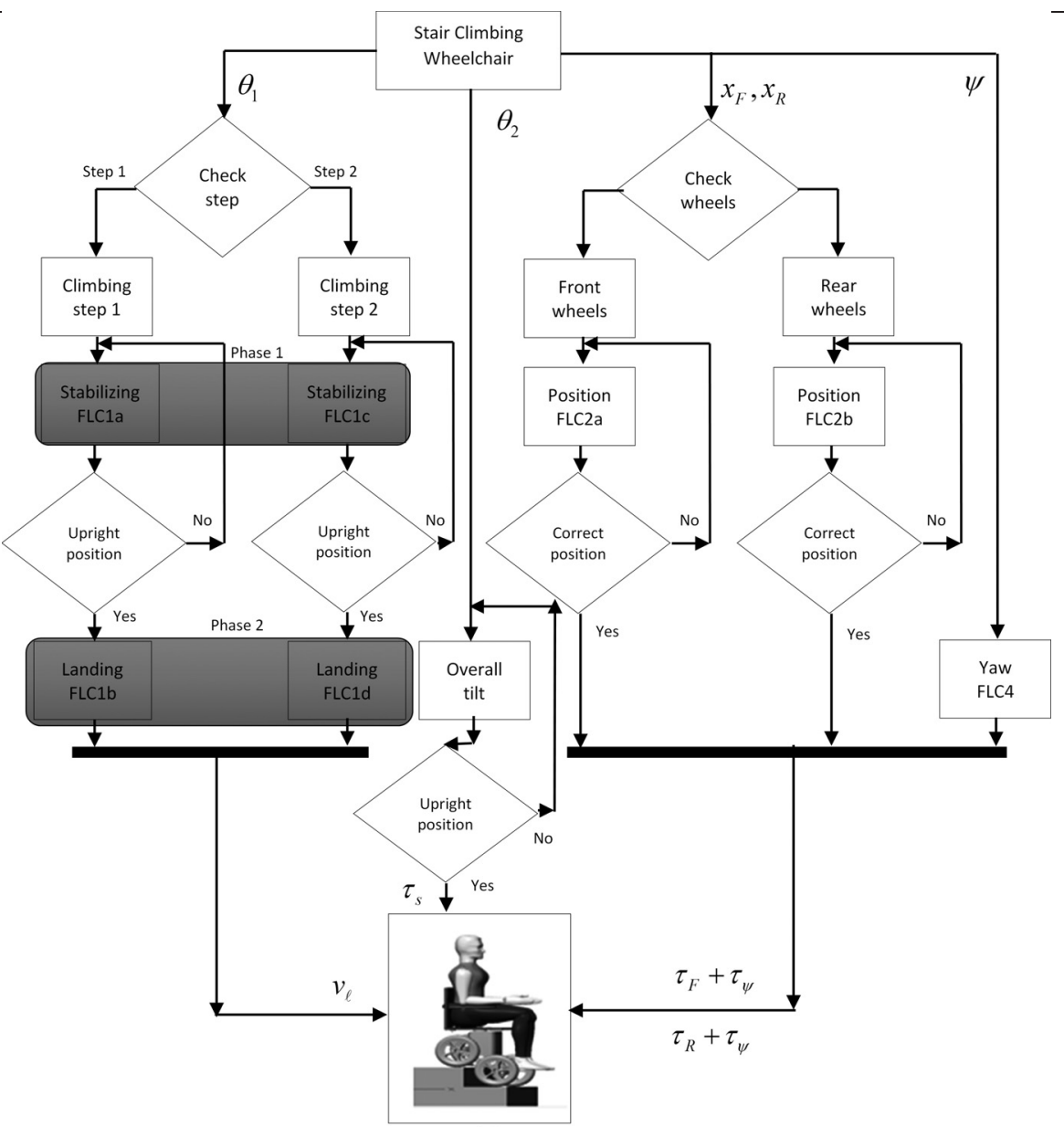

(a)

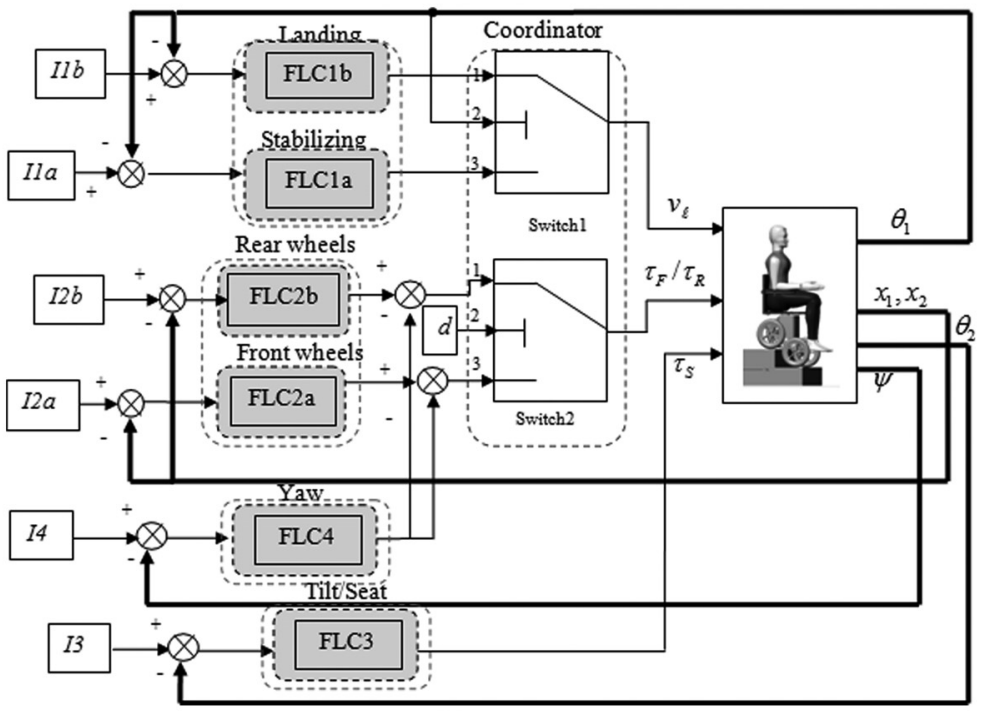

(b)

Fig. 5. Architecture of the modular fuzzy controller. (a) Flow chart of IPFL for $\tau_{F}$ and $\tau_{R}$; (b) Coordination of IPFL. 


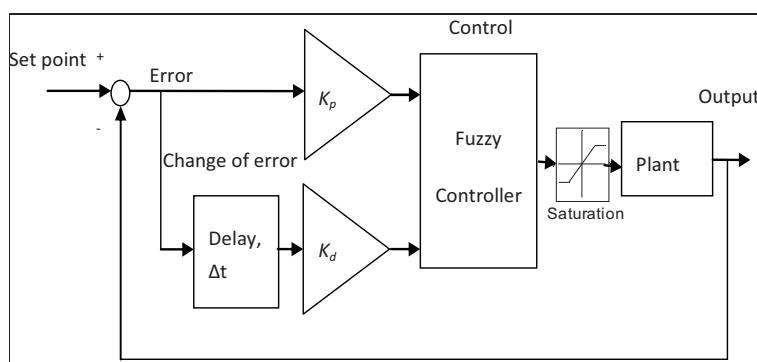

Fig. 6. Block diagram of a PD-fuzzy control system.

tively. A PD-Mamdani fuzzy controller is developed for this purpose to achieve steady and smooth system response. The main operation of the climbing process is initiated by lifting the link motor to the upright vertical (an inverted pendulum like) position while keeping the acting wheel motors at the same position as well as the tilt/seat stabilization to reach a certain angle, $\theta$. The control signals, $\tau$ and $v$ are defined as:

$$
\tau_{o r} v=k_{p} e+k_{d} \Delta e
$$

where $\tau$ or $v$ represents the control output signal, $k_{p}$ and $k_{d}$ are the proportional and differential gains respectively for the fuzzy gain scaling factors, $e$ is the input error and $\Delta e$ is the change of input error. The scaling factor constraints are known as sensitivity factors to the controller. The scaling factors may be tuned with heuristic trial and error approach. However, such an approach is tedious and consumes significant time for achieving favourable, and yet not optimum, results. Thus to avoid such issues these parameters are tuned with spiral dynamic optimization algorithm (SDA) [16] in this work. SDA is a nature-inspired optimization algorithm, which uses a metaheuristic strategy [11] similar to those employed in computational intelligence optimization algorithms [19].

For each FLC block, the error, $e$ and change of error, $\Delta e$ with corresponding gains are mapped to fuzzy inference to provide a control output, as defined by the fuzzy relational formulation:

$$
\tau_{o r} v=((e \wedge \Delta e)) \circ R
$$

Numerous methods can be acquired through fuzzy relations with the fuzziness mapping represented by $R$. Expert knowledge is used to develop fuzzy rules to minimize the angular position error for Link and tilt, and position of front and rear wheels.

In this work, Mamdani inference is the best option to be utilised as information about the system is limited.
Fuzzy Rules for link, $v_{\ell}$, wheels, $\tau_{F / R}$ and seat motors, $\tau_{S}$

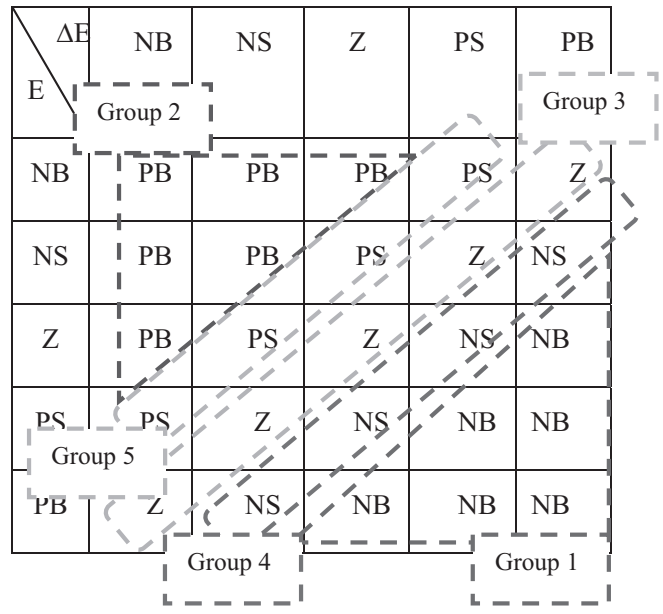

Sugeno type may be used if there is extensive system data and information available [14].

Mamdani type is thus utilized in all fuzzy controllers for the wheelchair in this work. Moreover, it is easy to understand by human expert and the rules are simple to formulate. The form of Mamdani type inference used is as follows:

\section{If $x$ is $A$ and $y$ is $B$ then $z$ is $C$}

The inputs are determined by $A$ and $B$ and the output for each linguistic variable set is indicated by $C$. Fuzzy values are obtained through fuzzification of the crisp values, and a defuzzification phase is realized for the fuzzy control output to achieve crisp control signal for application to the plant.

The fuzzy rules are developed based on expert knowledge to minimise the error and change of error of each input variable. All modules use the same fuzzy rule base and the fuzzy rules are shown in Table 2 [31]. There is a specific way to develop fuzzy rules set according to the system behaviour and desired performance. At first, the rules are viewed based on the most extreme situation as can be seen in Table 2. In general, if the error and the change of error are both positive big (PB), the control action must produce negative big (NB) signal in order to bring the output back to the reference set point as in Group 1. Similarly, the same amount of negative control action should be applied for the region within Group 1. On the contrary, if both the error and change of error are negative big (NB), positive big (PB) signal should be generated by the controller to ensure that the output is close to the set point. The same positive signal is produced for the 
region in Group 2. For the other two extreme points, if the error is positive big $(\mathrm{PB})$ and the change of error is negative big (NB) or vice versa, zero $(\mathrm{Z})$ control signal should be applied to the system because the system has reached a steady-state condition. The same amount of control signal is applied within the region in Group 3. Group 4 and Group 5 show that small amount of control action is needed to compensate for the errors. As the system performance varies, and this is problem dependent, the control signal is not necessarily fixed as discussed here. However, most control problems follow the standard rules and similar pattern as shown in Table 2 for the wheelchair system in this work.

The inputs used for the respective controllers are defined as follows: FLC1a and FLC1b take two inputs, which are angular position error of link, $e_{\theta_{1}}$ and change in angular position error of link/cluster $\Delta e_{\theta_{1}}$ respectively. The output is link velocity, $v_{\ell}$ within the universe of discourse. Similarly, FLC2a and FLC2b have input and output fuzzy relationship but in terms of position error and change in position error of front and rear wheels, $e_{x_{1} / x_{2}}$ and $\Delta e_{x_{1} / x_{2}}$ while the fuzzy output is front and rear wheel torques, $\tau_{F}$ and $\tau_{R}$. Error in angular position of tilt $e_{\theta_{2}}$ and change in angular position error of tilt, $\Delta e_{\theta_{2}}$ are the fuzzy input for FLC3 for the seat torque, $\tau_{S}$. The yaw error, $e_{\psi}$ and change of yaw error, $\Delta e_{\psi}$ are the fuzzy inputs to FLC4 for yaw torque compensation, $\tau_{\psi}$.

\subsection{Stair ascending}

In order to perform stair climbing operation, link motor, $v_{\ell}$ will rotate a link to the upright vertical position while the front wheel torque, $\tau_{F}$ keeps the left and right wheels in the same position. This is called stabilization phase where the link is stabilised at the upright position before continuing to the landing phase depending on the stair tread depth. This stabilization phase is designed to allow the wheelchair transformation from climbing mode to linear mode on the stairs. This will permit testing of variations of stairs' tread size for the climbing task and will allow the wheelchair to move in two-wheeled mode on the stairs. Once the rear wheels have landed on the next step, the rear wheel's torque, $\tau_{R}$ is activated and the front wheel's torque, $\tau_{F}$ is deactivated to ensure that the rear wheels are maintained at the same position on the stairs to prevent slipping. The link will repeat the stabilization phase and landing phase again and so forth for climbing operation to subsequent steps. It is found that both controllers ( $\mathrm{FLCl} 1$ and $\mathrm{FLC} 2$ ) need more specific tasks especially when switching from 'front to rear wheels torque' phases and from 'stabilizing to landing' phases and vice versa to perform stair climbing operation. This is due to the fact that all torques have specific tasks to ensure the stability of the system while upholding large payload mass (approximately $100 \mathrm{~kg}$ including human and wheelchair mass) on the stairs. This task is repeated automatically with the other motor, $\tau_{F}$ or $\tau_{R}$, to climb the next step and this process is characterized as a phase interchangeable mechanism: 'front and rear wheel' phases and 'stabilization and landing' phases. The two phases are interconnected to each other and require a complex control structure to supervise and coordinate the whole process.

A fuzzy controller block is added with sub modules, FLC1a and FLC1b to each stabilizing and landing phase module. Similar control structure is used for executing the front and rear wheel's torque but with additional task of linear motion and yaw angle control capability. The only difference is that the switching condition is based on the distance $d$ between wheels and stairs, and yaw control is added with the front or rear wheel's torque via FLC4 block. The front and rear wheel's torque allows the wheelchair to perform a linear motion in its two wheeled mode without any yaw movement on the stairs. Once it has reached the second step, the landing phase is activated in order to place the other pair of wheels on the stairs. The torque is switched accordingly and the set point inputs are represented by ' $I 2 a$ ' and ' $I 2 b$ ' for the front wheels phase and rear wheels phase respectively.

The stabilization of the whole system is realised with tilt/seat torque, $\tau_{s}$. The whole wheelchair system including humanoid is stabilized through FLC3 module via a constant $I 3$ throughout the stair climbing operation. The FLC3 controls the wheelchair seat to be levelled and is always active without the involvement of any switching action. The IPFL control structure has been designed and implemented for different situations. The main advantage of this design is that each module uses the same fuzzy rule to perform different control actions. Hence, only one FLC was designed and applied to achieve three different objectives, climbing, controlling the wheels position and the tilt angle.

\subsection{Safety precaution}

It is vital that the control structure is added with safety elements so as to account for the case of control failure due to any uncertainties or hard disturbance 
10 N.M. Abdul Ghani and M.O. Tokhi / Simulation and control of multipurpose wheelchair for disabled/elderly mobility

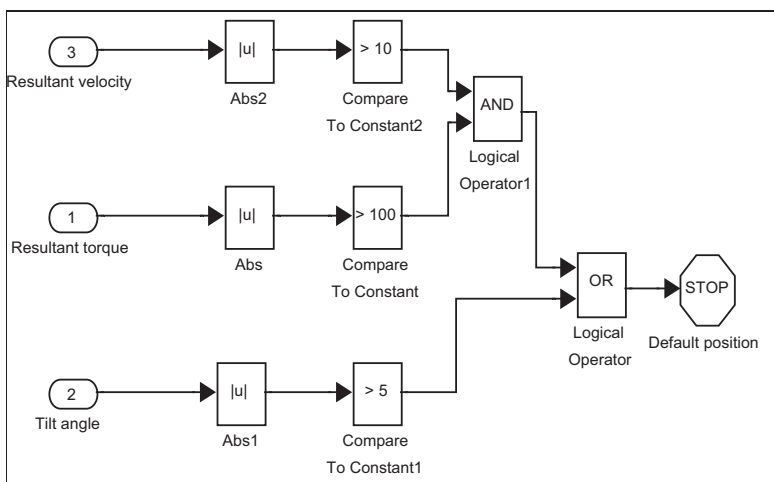

Fig. 7. Additional element for system safety.

force applied to the wheelchair system. It has been determined through tests that the system will become unstable if the torque and velocity are more than \pm $100 \mathrm{Nm}$ and $\pm 10 \mathrm{deg} / \mathrm{s}$ respectively. The tilt angle of the system must be within \pm 5 deg to make sure that the user stays within safe and stable region, almost upright position at all times. If the control signals are higher than certain amount of limits, the wheelchair system will stop automatically and transform to the default four-wheeled mode. The safety mechanism is shown in Fig. 7.

\subsection{Stair descending}

It is known that performing a descending operation is more challenging than ascending operation because there is no block/step at the front of the wheelchair to prevent the system from collapsing. Due to this situation, the wheels tend to roll forward and may slip off the stairs causing instability and thus making the system harder to control. In order to adapt to different system dynamics and descending motion, different set points and angular positions, $\theta_{1}$ are used. The main control structure for climbing down the stairs is similar to the climbing up task in automatic mode as mentioned earlier in the ascending task.

\subsection{Sit-to-stand and stand-to-sit}

A block diagram of the modular design is shown in Fig. 8 with integration between Simulink and VN4D and coordinated by switching elements. There are five modules to operate both sit-to-stand and standto-sit tasks, namely link/cluster lifting, height extension, back seat rotation, bottom seat rotation and overall tilt. A hybrid PD-Mamdani fuzzy controller is developed to perform smooth standing and sitting opera-

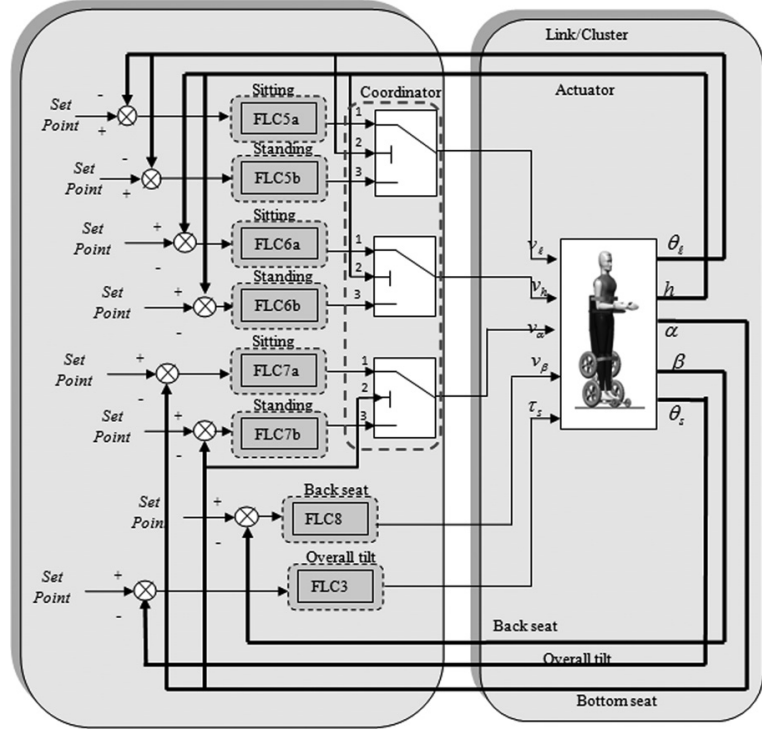

Fig. 8. Simulink/Matlab and VN4D integration.

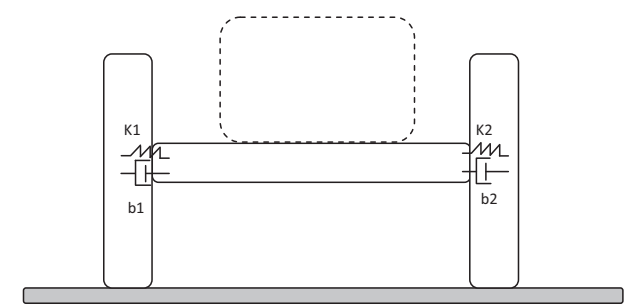

(a)

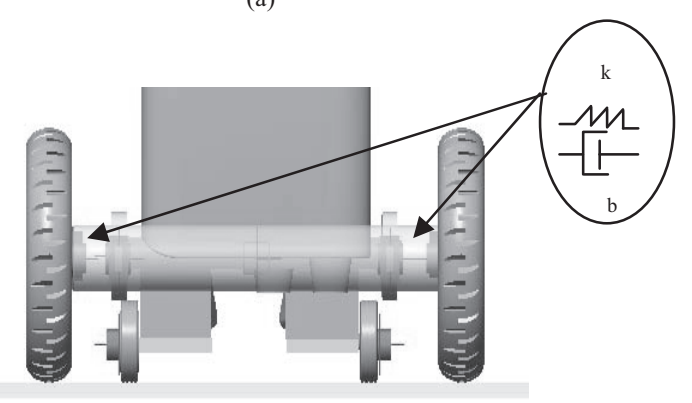

(b)

Fig. 9. Spring/Damping elements.

tions. The input for each modules is given in terms of set points. The set points for link/cluster lifting, height extension, back seat rotation, bottom seat rotation and overall tilt modules are $\theta_{\ell}, h, \beta, \alpha$ and $\theta_{s}$ respectively. These modules use fuzzy operation as indicated in the blocks; FLC5a, FLC6a and FLC7a for sit-to-stand task while blocks FLC5b, FLC $6 \mathrm{~b}$ and FLC7b for stand-tosit operation. Both tasks use the same FLC3 and FLC8 modules for back seat and overall tilt stabilization. 


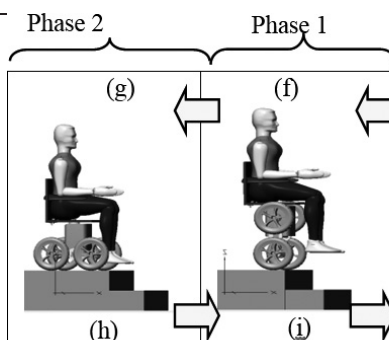

(h)

Phase $2 \quad$ Phase 1 Phase 2 Phase 1

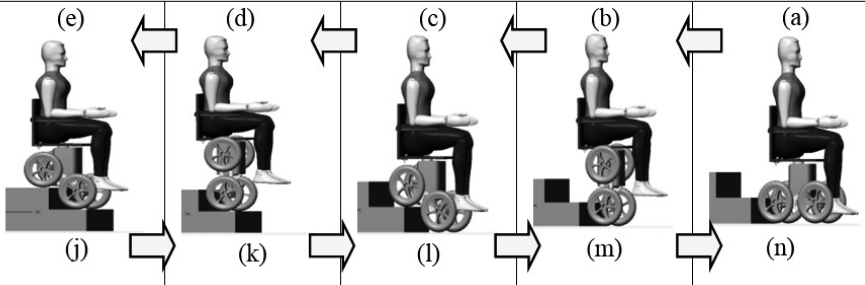

Fig. 10. Ascending and descending stairs motio.

Wheelchair with active suspension control can prevent traumatic shocks and vibration to the rider caused due to prolonged driving on rough outdoor surfaces, bumps and curbs. The rider can experience mental fatigue caused by the trauma which can worsen their health condition. By installing a suspension system, these problems can be alleviated and a highly comfortable ride achieved.

In this work, it is crucial to ensure smooth and good floor contact while performing stand-to-sit operation, referred to as back transformation. This is due to the system needing to support heavy load of the user and the mass of both rear wheels. The respective wheels may hit the ground with large impact in the absence of an appropriate mechanism. For this reason, all four wheels of the wheelchair system are independently mounted with revolute spring/damping element. This makes it easier to negotiate high impact and smoothen the back transformation process while maintaining good floor contact with the wheels. Figure 9 shows the location of revolute spring/damper as seen from the front view. The values of spring and damper constants used were $k 1=k 2=0.01 \mathrm{Nm} / \mathrm{deg}$ and $b 1=b 2=0.000914 \mathrm{Nms} / \mathrm{deg}$ respectively [4]. The damping elements are placed at both left and right side of the wheels with the same coefficient values.

\section{Simulation and performance analysis}

\subsection{Stair climbing}

The climbing operation was tested with two different stair depths, $d=0.302 \mathrm{~m}$ and $d=0.407 \mathrm{~m}$. Figure 10 shows visual images of the wheelchair performance while ascending Figs 10(a)-(g) and descending Figs 10(h)-(n) in VN4D. Both phases are repeated to ensure the stair climbing operation is done in sequence. The third, fourth and n-th steps will copy the same procedure as climbing the second step (not shown). Note that the wheelchair system climbs backwards up the stairs, with the user facing downstairs, and descends forward down the stairs with the user also facing downstairs as described in the ISO 7176-24: 2004 standard [9].

\subsubsection{Stair ascending}

Figures 11(a) and (b) illustrate simulation of the stair climbing performance according to the motion in Fig. 10 for both stair depths. It is noted that it took approximately $1.7 \mathrm{~s}$ and up to $4.5 \mathrm{~s}$ to complete the first and second steps respectively for the wheelchair to perform the stair climbing task for stair depth $d=$ $0.302 \mathrm{~m}$. The final step was completed in less than $3 \mathrm{~s}$, and thus it took the wheelchair approximately $7.3 \mathrm{~s}$ to reach the upper flat surface, as in Fig. 10(g). During climbing the first step, both the front motor torques are active while the rear are not active and vice versa as illustrated in Fig. 11(b). Note that the tilt/seat angle was able to stay at the upright position at all the times with seat motor torque $\tau_{s}$. For $d=0.407 \mathrm{~m}$, the system performed slower as there was a linear motion (red region) of the wheelchair on the second step as seen for both links during $2^{\text {nd }}$ step in Fig. 11(a). Both links remained at the same position while performing this linear task for almost $1 \mathrm{~s}$, from $3.8 \mathrm{~s}$ to $4.8 \mathrm{~s}$. The front and rear wheels (acting wheels) showed a travelled distance, $T_{d}$ of approximately $0.3 \mathrm{~m}$ during the linear motion (red region) process. The corresponding control efforts are shown in Fig. 11(b).

It is worth mentioning that the tilt angle was larger in previous work $[5,6]$ at the initial stage; this was $-25^{\circ}$ at Link 2, as compared to this new wheelchair configuration with only $-3^{\circ}$ for the initial tilt angular position. Moreover, the initial energies consumed by the cluster and tilt motors were much lower than previous wheelchair design $[5,6]$, and this has reduced by approximately $82 \%$ (from $110 \mathrm{Nm}$ to $20 \mathrm{Nm}$ ) for the wheel's torque and $69 \%$ (from $-160 \mathrm{Nm}$ to $-50 \mathrm{Nm}$ ) for the tilt motor torque in order to bring the wheelchair mechanism to the upright position. 

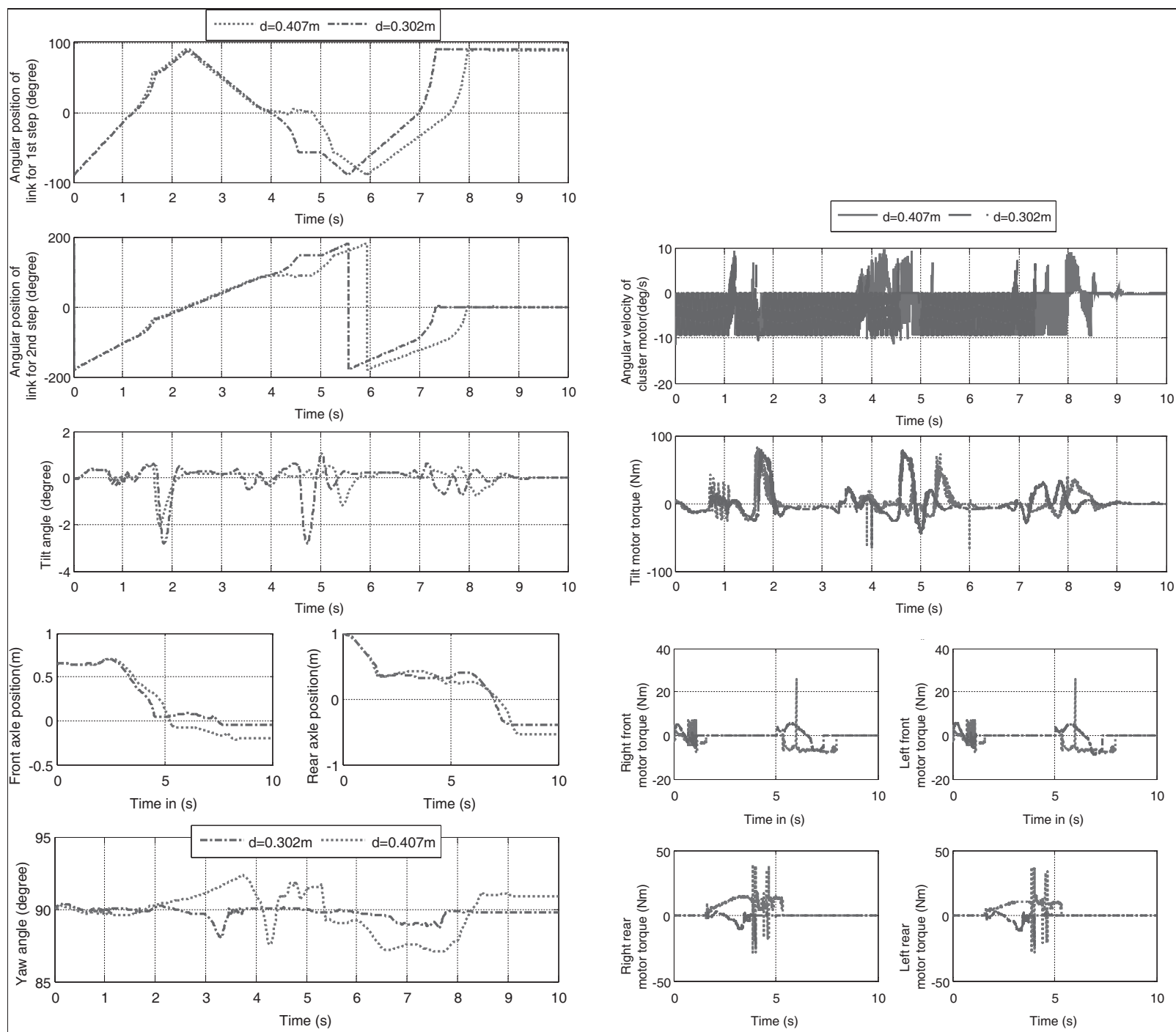

(a)
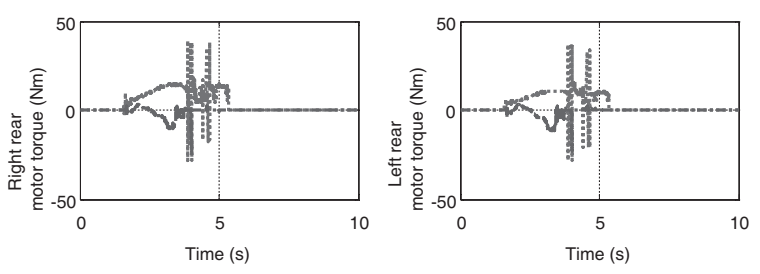

(b)

Fig. 11. (a) System performance for $d=0.302 \mathrm{~m}$; (b) Control input signals for $d=0.302 \mathrm{~m}$.

\subsubsection{Stair descending}

Contrary to ascending task, the transformation process involves stabilization in two wheeled mode via backward motion while forward stabilization is used in the descending operation as in Fig. 10(h)-(n). This is because the user/human needs to be at the same position as in ascending the stairs. Next, the system executes a landing phase to place the rear wheels to the next step from vertical position. Once the rear wheels are placed on the next step and the required distance is achieved, the operation is switched to the rear wheels motor control through 'front and rear wheel' switching phase.

For the descending task, similar test was conducted on different stair tread depths, $d=0.302 \mathrm{~m}$ and $d=$ $0.407 \mathrm{~m}$ as in the ascending task. The switching phases from Phase 1 (Stabilizing) to Phase 2 (Landing) can be seen clearly in this simulation and the performances are shown in Fig. 12. The performance is assessed with yaw and without yaw control on the respective wheels motor, due to the turning motion of wheels while in the descending operation. The steering motion will affect the system not to perform the descending task in straight-line manner and cause the wheels to turn either right or left, and this may cause the system to slip off the staircase and collapse.

As can be noticed in Fig. 12, the system was able to perform the descending task smoothly in a stable man- 

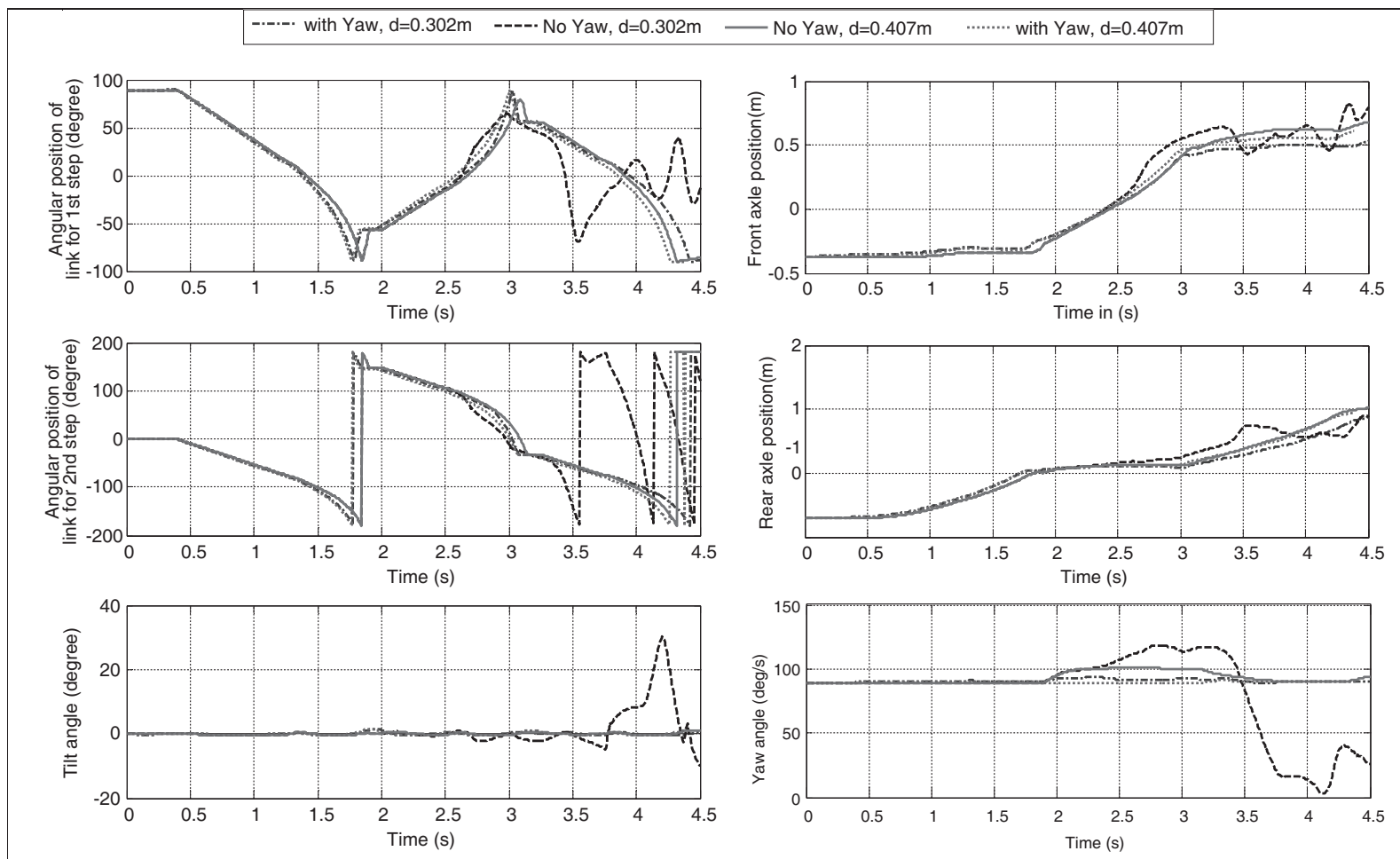

(a)
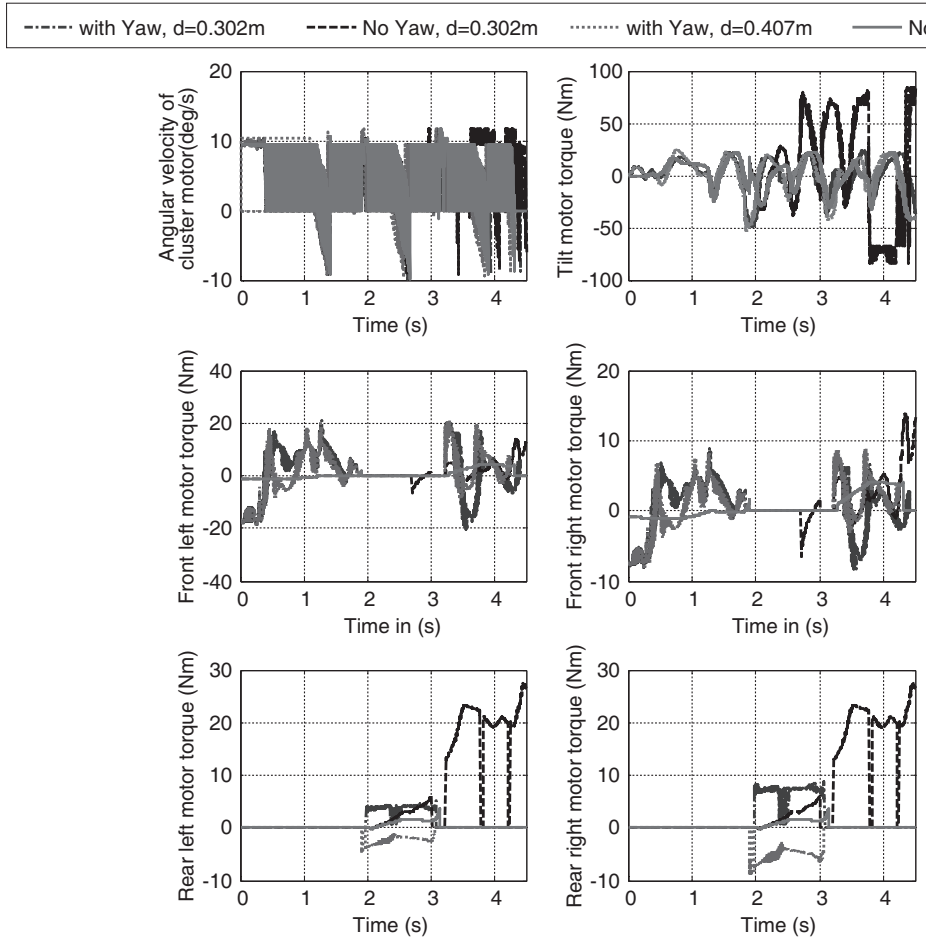

(b)

Fig. 12. (a) System performance with and without yaw control; (b) Control input signals with and without yaw control. 
ner with yaw control for both stair-tread depths. It is noted that the system performed better and was able to maintain the wheelchair straight without slipping and collapsing. The wheelchair on stair-tread depth, $d=$ $0.407 \mathrm{~m}$ also performed in similar manner in terms of the links angular position, tilt angle, respective wheels positions and yaw motion. As noted both wheels stayed on the stair during corresponding tasks as they moved within acceptable displacement while yaw and tilt angle resulted significant changes when climbing the second step but still in a stable manner.

It is obvious that the wheelchair system collapsed and became unstable after $3 \mathrm{~s}$ when tested with the stair-tread depth of $d=0.302 \mathrm{~m}$ without yaw control. This is due to the limited step space for the respective wheels to move and support the whole wheelchair system to perform the descending motion. It is also noticed that from two to three seconds, the yaw error was high for the system without yaw angle control on stairs tread depth of $0.407 \mathrm{~m}$ with approximately $10^{\circ}$ difference. The rear wheels motor was activated during this period to compensate for the error, thus producing negative motor torques to the rear wheels motor. As a result, the tilt angle for the seat was smaller for the system without yaw control as compared to the system with yaw control, $\pm 0.5^{\circ}$ and $\pm 1.5^{\circ}$ respectively. It is noted that it took the wheelchair approximately $1.8 \mathrm{~s}$ and up to $3 \mathrm{~s}$ to complete the first and second step manoeuvres respectively. The final step manoeuvre was completed in less than $1.5 \mathrm{~s}$, which was up to $4.3 \mathrm{~s}$ to reach on the lower flat surface according to Fig. 10(n). Both yaw and tilt angles exhibited large changes while the front and rear wheels were less affected when climbing the second and final steps for stair-tread size $d=0.302 \mathrm{~m}$.

\subsection{Sit-to-stand and stand-to-sit}

A displacement point of $4 \mathrm{~m}$ was tested to complete the linear motion task once the human was in fully standing mode in the upright position. The performance was assessed with two approaches; with spring/damper and without spring/damper mechanism. Note that the wheelchair started to travel in linear forward motion to $4 \mathrm{~m}$ once it had reached vertical standing position at approximately $2 \mathrm{~s}$ after performing sitto-stand operation. It took less than $4 \mathrm{~s}$ to travel to the set point and stayed until $7 \mathrm{~s}$. It is also noted that both back seat and overall tilt angles were kept at zero degrees although the tilt angle produced was slightly higher in magnitude. This is due to the fact that the
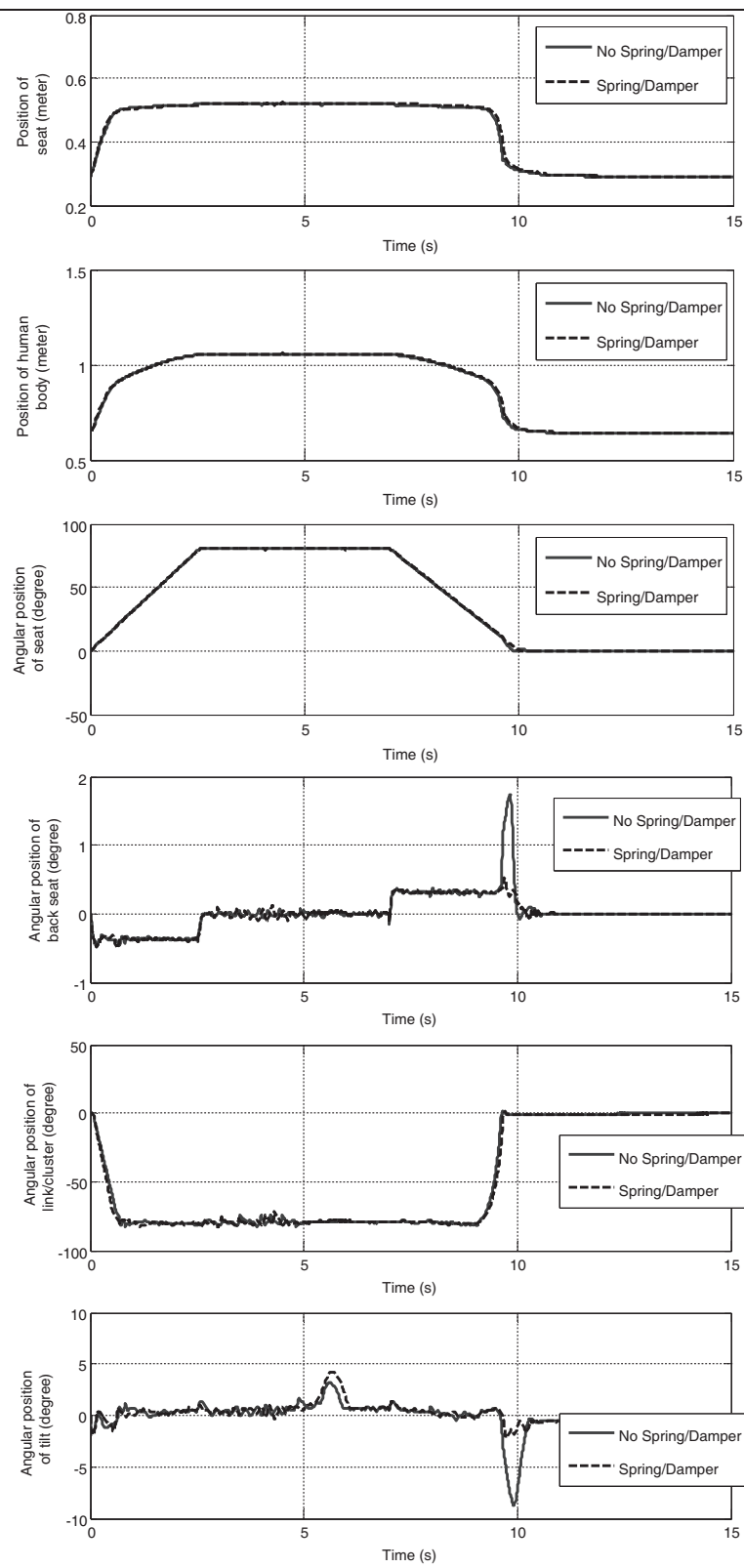

Fig. 13. System performances.

wheelchair was about to stop at $4 \mathrm{~m}$ and counteracted the forward motion by giving opposite torque to the wheels.

For sit-to-stand, both approaches showed similar performances. It is noted that it took less than $1 \mathrm{~s}$ for the cluster/link to lift the wheelchair to the upright position, $-80^{\circ}$ and approximately $2.5 \mathrm{~s}$ to complete the transformation process and reach the standing position. The seat angle was fully unfolded at $80^{\circ}$ and lifted to $0.51 \mathrm{~m}$ height. During these operations, the tilt/seat an- 

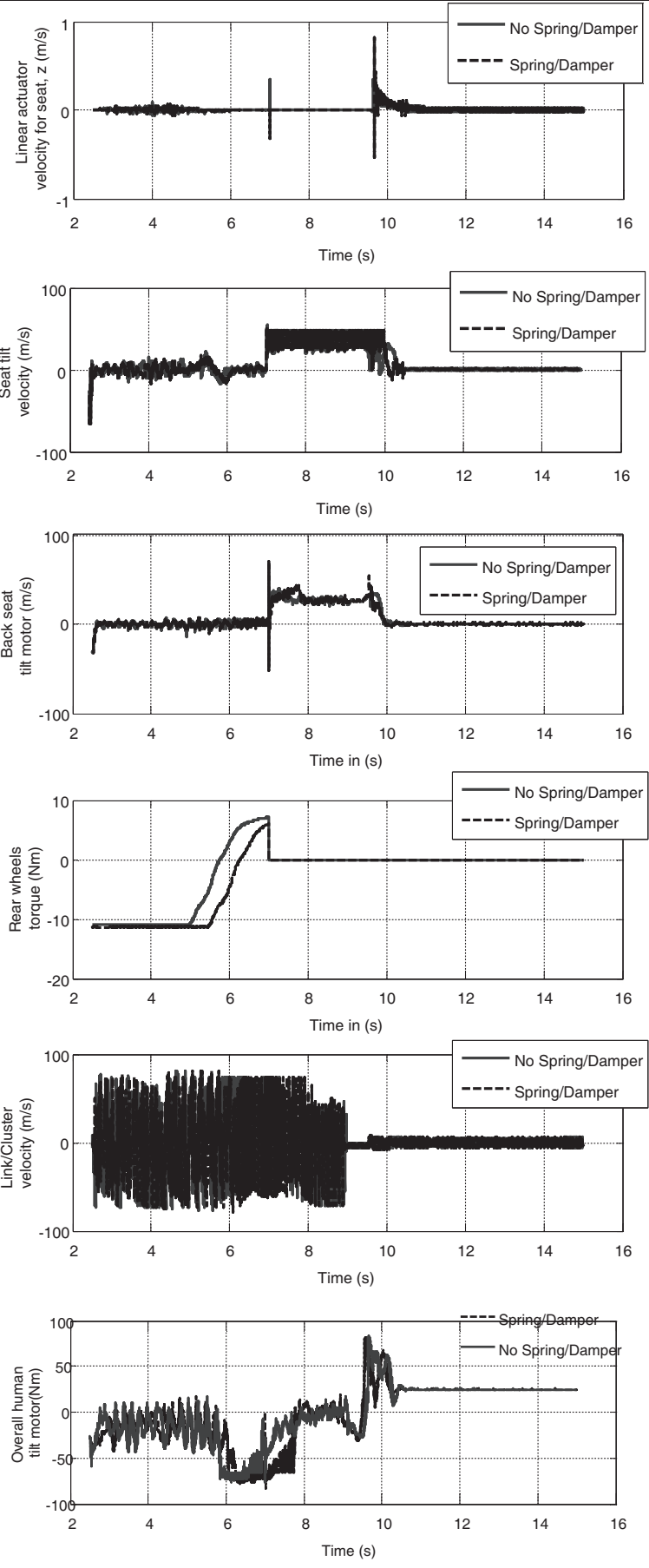

Fig. 14. Control efforts.

was activated and transformed the wheelchair from two-wheeled to four-wheeled mode (as shown in the graph).

The stand-to-sit operation took $3 \mathrm{~s}$ to bring the respective wheels on the ground in a stable manner even though there was a big change in the tilt angular position during the landing process. Similar performance was noted with the back seat motion; reached up to $1^{\circ}$ for the system without spring/damper mechanism. However, both tilt and back seat angles were significantly reduced by more than $50 \%$ as compared to the system without spring/damper mechanism. The corresponding control velocity and torque are shown in Fig. 14. Note that there was an impulse as indicated at $7 \mathrm{~s}$ at which the back transformation process occurred.

It is noted that both angular position of back seat and tilt were quite high without spring/damper mechanism due to fast landing operation. However, the force exerted by the pair of spring/damper mechanisms characterized by $k 1, b 1$ and $k 2, b 2$ were reduced. The passive control approach used for the transformation process showed the significant ability of the approach to suppress high ground impact and ensure user's comfort. The results presented have shown that the developed wheelchair model is able to perform multiple tasks: stair climbing (ascending/descending) and sit-to-stand/stand-to-sit operations with a humanoid model of $71 \mathrm{~kg}$ weight in a stable manner with reduced initial torque and reduced tilt angle.

\section{Conclusion}

A new reconfigurable wheelchair using link/cluster rotation with compact design has been developed in VN4D environment. The introduced system is intended for use in small and confined indoor spaces for disabled and elderly mobility to enable them perform daily life activities independently. An interchangeable phase modular fuzzy logic control mechanism has been proposed and successfully implemented with the wheelchair system to perform two tasks: 'balancing and stabilizing' and 'front and rear' wheels switching. The same wheelchair system is also able to perform stair climbing and sit-to-stand/stand-to-sit tasks. The stabilizing mechanism in a two-wheeled position has been utilised for the sit-to-stand transformation for saving space. A chair height extension mechanism and several revolute motors have been added to accomplish the standing motion in vertical straight position. A passive suspension system has been developed and incor- 
porated into the wheelchair by adding spring/damping element at each wheel to provide a comfortable ride to the user especially during landing in the stand-tosit process. It has been demonstrated that the system performs well with damping mechanism; more than $70 \%$ and $38 \%$ reduction for the $4 \mathrm{~m}$ and $5 \mathrm{~m}$ travel distances respectively in tilt and back seat angles have been achieved. It has been demonstrated that, the current wheelchair configuration produces low initial tilt angle and low overall tilt torque; approximately more than $50 \%$ reduction as compared to previous design, for transformation to the upright two-wheeled mode.

This work has carried out a first phase of the project study in simulations on modelling and control of a multipurpose wheelchair for disabled/elderly with reduced initial torque and reduced tilt angle. A range of standard daily life activities have been tested using the wheelchair system to validate the developed control approach. The results achieved are convincing and thus suitable for real hardware implementation. Further work will look at robustness of the control mechanism with humans of greater weight, and realisation of the proposed design and control approach.

\section{References}

[1] S.C. Abdulla, O. Sayidmarie and M.O. Tokhi, Functional electrical stimulation-based cycling assisted by flywheel and electrical clutch mechanism: A feasibility simulation study, Robotics and Autonomous Systems 61 (2013), 443-472.

[2] H. Adeli and S.L. Hung, Machine Learning - Neural Networks, Genetic Algorithms and Fuzzy Systems, John Wiley and Sons, New York, 1995.

[3] H. Adeli and K. Sarma, Cost Optimization of Structures Fuzzy Logic, Genetic Algorithms and Parallel Computing, John Wiley and Sons, West Sussex, United Kingdom, 2006.

[4] S. Ahmad, M.O. Tokhi and N.H. Siddique, Modular fuzzy control with input shaping technique for transformation of two-wheeled wheelchair to four-wheeled mode, Proceeding of IEEE Industrial Electronics and Applications (ISIEA), (2010), 562-566

[5] S. Ahmad, N.H. Siddique and M.O. Tokhi, A modular fuzzy control approach for two-wheeled wheelchair, Journal of Intelligent Robotic System (2011), 401-426.

[6] S. Ahmad, N.H. Siddique and M.O. Tokhi, Modelling and simulation of double-link scenario in a two-wheeled wheelchair, Integrated Computer Aided Engineering 21 (2014), 119-132.

[7] S. Ahmad and M.O. Tokhi, Forward and backward motion control of wheelchair on two wheels, Proceeding of IEEE Conference on Industrial and Applications, (ICIEA), (2008), 461-466.

[8] A.M. Almeshal, K.M. Goher and M.O. Tokhi, Dynamic modelling and stabilization of a new configuration of two-wheeled machines, Robotics and Autonomous Systems 61(5) (2013), $443-472$.
[9] Canadian Centre for Occupational Safety and Health, 2010 (28 ${ }^{\text {th }}$ Nov 2012), [Available from], http://www.ccohs.ca/oshanswers/safety_haz/stairs_fallprevention.html.

[10] C. Cardero, Articulated human body, 2012, https://grabcad com/library/articulated-human-body- 2 .

[11] L.F.S. Coletta, E.R. Hruschka, A. Acharya and J. Ghosh, Using metaheuristics to optimize the combination of classifier and cluster ensembles, Integrated Computer-Aided Engineering 22(3) (2015), 229-242.

[12] R.A. Cooper, M.L. Boninger, R. Cooper and A. Kelleher, Use of the independence 3000 IBOT transporter at home and in the community: A case report, Disability and Rehabilitation: Assistive Technology 1-2 (2006), 111-117.

[13] D. Ding and R.A. Cooper, Electric powered wheelchairs, a review of current technology and insight into future directions IEEE Control Systems Magazine 25 (2005), 22-34.

[14] A.P. Engelbrecht, Computational intelligence: An introduc tion, Chichester, England, John Wiley and Sons, 2007.

[15] B. Fariba, R. Robert, J.M. Parviz and S. Gunther, Biomechan ical analysis of sit-to-stand transfer in healthy and paraplegic subjects, Clinical Biomechanics 15 (2000), 123-133.

[16] N.M.A. Ghani, A.N.K. Nasir and M.O. Tokhi, Optimization of fuzzy logic scaling parameters with spiral dynamic algorithm in controlling a stair climbing wheelchair: Ascending task, Proceeding of $19^{\text {th }}$ International Conference on Meth ods and Models in Automation and Robotics (MMAR) (2014) 776-781.

[17] K.M. Goher, Modelling and simulation of a reconfigurable wheelchair with sit-to-stand facility for a disabled child, Pro ceeding of IEEE $18^{\text {Th }}$ International Conference on Methods and Models in Automation and Robotics, (MMAR), (2013), 430-434.

[18] J. Huang, F. Ding, T. Fukuda and T. Matsuno, Modelling and velocity control of a novel narrow vehicle based on mobile in verted pendulum, IEEE Trans on Control Systems Technology 21(5) (2013), 1607-1617.

[19] J. Chen and T. Wu, A computational intelligence optimization algorithm: Cloud drops algorithm, Integrated Computer Aided Engineering 21(2) (2014), 177-188.

[20] J. James and J.C. Abbas, Gillaette, using electrical stimulation to control standing posture, IEEE Control System Magazines (2001), 80-90.

[21] Johnson and Johnson Company, Independence iBOT mobil ity system, (1 $1^{\text {st }}$ Jun 2012), [Available from], http://www.ibotnow.com/index.html.

[22] F.M. Juan-Antonio, G. Cipriano and G. Javier, Assistive nav igation of a robotic wheelchair using a multi hierarchical model of the environment, Integrated Computer Aided Engi neering 11(4) (2004), 309-322.

[23] R. Kamnik and T. Bajd, Standing-up robot: An assistive rehabilitative device for training and assessment, Journal of Med ical Engineering and Technology 28(2) (2004), 74-80.

[24] M. Lawn and T. Takeda, Design of a robotic-hybrid wheelchair for operation in barrier present environments, Proceeding of IEEE International Conference on Medicine and Biology Society (1998), 2678-2681.

[25] M.J. Lawn, T. Sakai, M. Kuroiwa and T. Ishimatsu, Development and practical application of a stair climbing wheelchair in nagasaki, Journal of HWRS-ERC 2 (2001), 33-39.

[26] Z. Li, C. Yang, C. Su and W. Ye, Adaptive fuzzy-based motion generation and control of mobile, Engineering Applications of Artificial Intelligence 30 (2014), 86-95.

[27] R. Morales, V. Feliu and A. González, Optimized obstacle avoidance trajectory generation for a reconfigurable staircase 
climbing wheelchair, Robotics and Autonomous Systems $\mathbf{5 8}$ (2010), 97-114.

[28] MSC software simulating reality, delivering certainty, MSC nastran multidisplinary structure analysis, http://www.mscsoftware.com/product/msc-nastran.

[29] S. Nakajima, Proposal for step-up gait of RT-mover, a fourwheel-type mobile robot for rough terrain with simple leg mechanism, Proceeding of IEEE International Conference on Robotics and Biomimetics (2010), 351-356.

[30] I. Oden and E. Knutsson, Evaluation of the effects of muscle stretch and weight load in patients with spastic paraplegia, Scan J Rehabil Med 13(4) (1981), 117-121.

[31] M.D. Philippa and K. Andrew, Frequency of the sit to stand task: An observational study of free-living adults, Applied Ergonomics 41 (2010), 58-61.

32] R. Ping, M. Chan, K.A. Stol and C.R. Halkyard, Review of modelling and control of two-wheeled robots, Annual Reviews in Control 37(1) (2013), 89-103.

[33] G. Quaglia, W. Franco and R. Oderio, Wheelchair, q, a mechanical concept for a stair climbing wheelchair, Proceeding of IEEE International Conference on Robotics and Biomimetics, Guilin, China, (2009), 800-805.

[34] G. Raju, J. Zhou and R.A. Kisner, Hierarchical fuzzy control, International Journal of Control 54(5) (1991), 1201-1216.

35] G.G. Rigatos, Adaptive fuzzy control for differentially flat MIMO nonlinear dynamical systems, Integrated Computer Aided Engineering 20(2) (2013), 111-126.

36] N. Siddique and H. Adeli, Computational intelligence - synergies of fuzzy logic, Neural Networks and Evolutionary Computing, Wiley, West Sussex, United Kingdom, 2013.

[37] Y. Sugahara, N. Yonezawa and K. Kosuge, A novel stairclimbing wheelchair with transformable wheeled four-bar linkages, Proceeding of IEEE/RSJ International Conference on Intelligent Robots and Systems (2010), 3333-3339.

[38] Z. Sun, N. Wang and Y. Bi, Type-1/Type-2 fuzzy logic systems optimization with RNA genetic algorithm for double inverted pendulum, Appl Math Modell 39(1) (2014), 70-85.

[39] Sunwa Co. Ltd (2006). (1 ${ }^{\text {st }}$ Apr 2012), [Available from], http: //www.sunwa-jp.co.jp/en/accessibility/.

[40] M.K.A. Tareq, Design and development of a new stabilization mechanism for two-wheeled wheelchair, Master's Thesis, International Islamic University of Malaysia, 2015.

41] Y. Teng, T. Wang, C. Yao and X. Li, The research of tension optimal estimation and stair-climbing ability of transformation wheelchair robot, Proceeding of $29^{\text {th }}$ Chinese Control Conference (2010), 3716-3721.

[42] Top Chair, (2003). The first stair-climbing powered wheelchair, (1 ${ }^{\text {st }}$ Apr 2012), [Available from], http://www.topchair. fr/.
[43] J. Vascak, Fuzzy control of a physical double inverted pendu lum, computational intelligence, Lecture Notes in Computer Science (1625) (1999), 482-494.

[44] L. Vermeiren, A. Dequidt and T.M. Guerra, Modeling, control and experimental verification on a two-wheeled vehicle with free inclination: An urban transportation system, Contro Engineering Practice 19 (2011), 744-756.

[45] S.L. Wang, Motion simulation with working model 2D and MSC, visual nastran 4D, J Comput Soft Engin 1(2) (2001), 193-196.

[46] Wheelchair-part 19: Wheeled mobility devices for use as seats in motor vehicles, ISO 7176-19, 2008. http://wwwiso.org/iso/ home/store/catalogue_ics/catalogue_detail_ics.htm?csnumbe $\mathrm{r}=40993$.

[47] Wheelchair-part 24: Requirements and test methods for useroperated stair-climbing devices, ISO 7176-24, 2004. http:// www.iso.org/iso/home/store/catalogue_ics/catalogue_detail_ ics.htm?csnumber $=45653$.

[48] D.A. Winter, Biomechanics and motor control of human movement, Wiley-Interscience, New York, 1990.

[49] J. Xu, Z. Guo and T. Heng, Synthesized design of a fuzzy logic controller for an underactuated unicycle, Fuzzy Sets and Systems 207 (2012), 77-93.

[50] W.Y. Hsu, Assembling a multi-feature EEG classifier for leftright motor data using wavelet-based fuzzy approximate entropy for improved accuracy, International Journal of Neural Systems 25(8) (2015).

[51] L. Forero Mendoza, M. Vellasco and K. Figueiredo, Intelligent multiagent coordination based on reinforcement hierarchical neuro-fuzzy models, International Journal of Neural Systems 24(8) (2014), 1450031.

[52] C. Chai and Y.D. Wong, Fuzzy cellular automata models for signalized intersections, Computer-Aided Civil and Infras tructure Engineering 30(12) (2015), 951-964.

[53] J.L. Ponz-Tienda, E. Pellicer, J. Benlloch-Marco and C. Andrés-Romano, Fuzzy project scheduling problem with minimal generalized relations, Computer-Aided Civil and Infrastructure Engineering 30(11) (2015), 872-891.

[54] W. Zhang, K. Sun, C. Lei, Y. Zhang, H. Li and B.F. Spencer, Jr., Fuzzy analytic hierarchy process synthetic evaluation models for the health monitoring of shield tunnels, ComputerAided Civil and Infrastructure Engineering 29(9) (2014) $676-688$.

[55] H. Adeli and S.L. Hung, A fuzzy neural network learning model for image recognition, Integrated Computer-Aided Engineering 1(1) (1993), 43-55.

[56] X. Jiang and H. Adeli, Fuzzy clustering approach for accu rate embedding dimension identification in chaotic time se ries, Integrated Computer-Aided Engineering 10(3) (2003) 287-302. 